\section{Praxisbesonderheiten im Arzneimittelregressverfahren}

Procedures in Justifying Drug Prescription

\section{Zusammenfassung}

Die Wirtschaftlichkeit der Arzneimittelverordnung in der gesetzlichen Krankenversicherung wird durch Prüfgremien überwacht. Im Richtgrößenprüfverfahren droht dabei der Arznei-mittelregress, wenn das Richtgrößenvolumen überschritten wird und diese Überschreitung nicht durch Praxisbesonderheiten gerechtfertigt ist. Nur durch eine sorgfältige Dokumentation lassen sich Praxisbesonderheiten erfolgreich nachweisen. Die Besonderheiten bei Arzneimittelinnovationen und die regional unterschiedlichen Prüfvereinbarungen/Richtgrößenvereinbarungen erfordern besondere Beachtung.
Abstract

The efficiency of drug prescription in the (German) system of Statutory Health Insurance is monitored by boards of inquiry. A doctor may be liable if the total amount of money spent on drugs he prescribed exceeds the cost guidelines, unless these costs are due to/caused by distinctive features of his practice. To prove distinctive features, meticulous documentation is required. Attention should be given to special problems regarding innovative drugs and the regional differences between the procedural rules and cost guidelines.
Diagnostik und Therapie, und damit auch die Pharmakotherapie, müssen im Rahmen der gesetzlichen Krankenversicherung dem Wirtschaftlichkeitsgebot entsprechen. Das Gesetz verlangt, dass die Leistungen ausreichend, zweckmäßig, nicht mehr als notwendig und wirtschaftlich sind. Gleichzeitig haben sie dem allgemein anerkannten Stand der medizinischen Erkenntnisse zu entsprechen und den medizinischen Fortschritt zu berücksichtigen. Der Leistungsanspruch des Patienten ist nicht auf die preisgünstigste Versorgung beschränkt. Das Recht fordert allerdings, dass bei therapeutisch gleichwertigen Behandlungsalternativen der Preis zu berücksichtigen ist.

\section{Prüfverfahren}

Für den niedergelassenen Vertragsarzt gehören die sog. „Wirtschaftlichkeitsprüfungen“ häufig bereits zum Praxisalltag. Der Gesetzgeber hat mit den jüngsten Gesundheitsreformen für den
Bereich der Pharmakotherapie die sog. Richtgrößenprüfung zur Prüfungsmethode der ersten Wahl gemacht und der zuvor am weitesten verbreiteten statistischen Vergleichsprüfung die Bedeutung genommen.

Grundlage der Richtgrößenprüfung sind abstrakte, fallbezogene Verordnungsbeträge pro Quartal für Arznei-, Verband- und Heilmittel. Sie werden arztgruppenspezifisch und unter Berücksichtigung von Altersgruppen oder gesondert für Mitglieder und Familienangehörige einerseits und Rentner andererseits regional zwischen KV und den Spitzenverbänden der Krankenkassen vereinbart. Die Richtgrößen sind keine Höchstgrenzen für die individuelle Verordnung, sondern Indikator für Wirtschaftlichkeit/ Unwirtschaftlichkeit im Rahmen eines Richtgrößenprüfverfahrens. In $§ 106$ Abs. 5a Satz 3 SGB V ist geregelt, dass bei einer Überschreitung des Richtgrößenvolumens um mehr als 25\% vom Vertragsarzt nach Feststellung durch den Prüfungsausschuss der sich daraus ergebende Mehraufwand den Kranken- 
kassen nur zu erstatten ist, soweit dieser nicht durch Praxisbesonderheiten begründet ist. Damit kommt der Definition, der Darstellung und der Quantifizierung von Praxisbesonderheiten die entscheidende Bedeutung im Richtgrößenprüfverfahren zu.

\section{Praxisbesonderheiten}

Für die statistische Vergleichsprüfung gelten nach gefestigter Rechtsprechung solche Umstände als Praxisbesonderheiten, die sich auf das Verordnungsverhalten des Arztes auswirken und in den Praxen der Vergleichsgruppe typischerweise nicht oder nicht in derselben Häufigkeit anzutreffen sind [1]. Die geltend gemachten Besonderheiten müssen also entweder für die Arztpraxen der Vergleichsgruppe atypisch sein oder von ihrer Häufigkeit her so wesentlich über ihrem durchschnittlichen Anteil in den Praxen der Vergleichsgruppe liegen, dass allein ihre große Zahl im Ergebnis ein spezifisches Qualitätsmerkmal der betreffenden Arztpraxis darstellt [2]. Diese Definition der Praxisbesonderheiten lässt sich auf die Richtgrößenprüfung übertragen. Denn obwohl die fachgruppenspezifische „Richtgröße“ vertraglich vereinbart ist, stellt sie doch einen fachgruppenbezogenen Durchschnittswert dar [3]. Daher kann auch bei der Richtgrößenprüfung bei Überschreitung des Richtgrößenvolumens eine Unwirtschaftlichkeit nur angenommen werden, wenn die wesentlichen Leistungsbedingungen (Zusammensetzung der Patientenklientel) des geprüften Arztes mit den wesentlichen Leistungsbedingungen der Ärzte der Fachgruppe übereinstimmen. Da die Richtgröße auf eine durchschnittliche Patientenstruktur der Fachgruppe berechnet wurde, ist also deren Überschreitung gerechtfertigt, wenn eine von der Regelpraxis abweichende $\mathrm{Zu}-$ sammensetzung des Patientengutes oder/und eine besondere Praxisausrichtung nachgewiesen werden kann. Nach den Gesetzmaterialien zum GKV-Gesundheitsreformgesetz 2000 [4] kommt daher als Praxisbesonderheit insbesondere ein hoher Anteil von Patienten in Betracht, deren Versorgung mit einem extrem hohen Aufwand an Arzneimitteln verbunden ist.

Der erfolgreiche Nachweis solcher Praxisbesonderheiten erfordert

- die Identifikation der Praxisbesonderheiten,

- die Darstellung der Besonderheiten,

- die Darstellung des Mehraufwandes im Einzelfall und

- die Extrapolation der Mehrkosten des Einzelfalles auf den Mehraufwand der Gesamtpraxis.

Dabei lässt sich der „Mehraufwand“ nur im Vergleich mit dem Durchschnitt der Fachgruppe quantifizieren. In der Praxis ist dies häufig schwierig, weil entsprechende Daten zum Fachgruppendurchschnitt nicht vorliegen. Hilfreich können hier die Erhebungen des Zentralinstituts für die kassenärztliche Versorgung in der Bundesrepublik Deutschland (www.zi-berlin.de) sein. Hier finden sich u.a. Angaben zur durchschnittlichen Häufigkeit bestimmter Diagnosen in der jeweiligen Fachgruppe. So kann beispielsweise nach Analyse des Anteiles der Psoriasispatienten in der eigenen Praxis im Vergleich mit dem durchschnittlichen Anteil in der Fachgruppe nachgewiesen werden, dass diese Patientengruppe überdurchschnittlich häufig in der eigenen Praxis angetroffen wird.

\section{Dokumentation}

Der Nachweis von Praxisbesonderheiten erfordert aber in jedem Fall, dass die Verordnung konsequent mit der zugehörigen Indikation dokumentiert wird. Eine gute Dokumentation beinhaltet auch die jeweiligen Kosten und ermöglicht es, zum Quartalsende die in den teuren Indikationen aufgelaufenen Verordnungskosten versichertenbezogen zuzuordnen und aufzustellen. Dabei sei beachtet, dass der Arzt ohnehin sozial- und zivilrechtlich zur Dokumentation verpflichtet ist, wobei der Umfang der Dokumentationspflicht vom Zweck der Therapiesicherung, der Beweissicherung und der Rechenschaftslegung her zu definieren ist. Eine weitergehende Dokumentation zur Regressprophylaxe mag zwar sozial- und zivilrechtlich nicht geschuldet sein. Aufgrund der Mitwirkungspflicht im Wirtschaftlichkeitsprüfverfahren trägt aber der Verordner das Risiko, ohne entsprechende Dokumentationen die Prüfinstanzen nicht von der Wirtschaftlichkeit seines Handelns überzeugen zu können.

Durch eine regelmäßige Dokumentation kann der Arzt unter Umständen auch dazu beitragen, dass ein Prüfverfahren erst gar nicht eingeleitet wird, denn einige kassenärztliche Vereinigungen wünschen sogar eine quartalsweise Meldung der Praxisbesonderheiten, da sie dann die Möglichkeit haben, die Überschreitungen des Richtgrößenvolumens vor Einleiten einer Wirtschaftlichkeitsprüfung zu verifizieren.

\section{Kompensatorische Einsparungen}

Während die Rechtsprechung zur statistischen Vergleichsprüfung neben den Praxisbesonderheiten im dargestellten Sinne auch jeweils die so genannten „kompensatorischen Einsparungen“ berücksichtigte, finden sich diese ausdrücklich nicht in den gesetzlichen Regelungen zur Richtgrößenprüfung. Dennoch sind diese gleichfalls zu berücksichtigen [5]. Auch in der Bundesempfehlung zu Richtgrößen vom 21. 2. 2000 hatten die Partner der gemeinsamen Selbstverwaltung auf Bundesebene kompensatorische Einsparungen im Richtgrößenprüfverfahren als berücksichtigungsfähig anerkannt. Damit kann also wie bisher auch im Richtgrößenprüfverfahren ein hohes Verordnungsvolumen durch kompensatorischen Minderaufwand in anderen Leistungsbereichen gerechtfertigt werden. Nach der Rechtsprechung des BSG muss der Arzt allerdings das Vorliegen von Einsparungen, den methodischen Zusammenhang mit dem Mehraufwand, die medizinische Gleichwertigkeit und die kostenmäßigen Einsparungen strukturell darlegen. Er darf sich nicht darauf beschränken, sich auf sein Vorbringen zu früheren Quartalen zu beziehen, sondern muss konkret für den jeweiligen Prüfungszeitraum vortragen. Ein kompensatorischer Minderaufwand kann insoweit beispielsweise in unterdurchschnittlichen Überweisungen, unterdurchschnittlichen Krankenhauseinweisungen oder unterdurchschnittlichen AU-Tagen bestehen. 
Da die Richtgröße jeweils für ein Folgejahr vereinbart wird und Arzneimittelinnovationen damit denklogisch in der Richtgröße nicht erfasst sein können, müssten auch solche als Praxisbesonderheit anerkannt werden. Die Überschreitung der Richtgröße ist gerechtfertigt und erlaubt nicht den Schluss auf unwirtschaftliche Verordnungsweise, wenn die Überschreitung durch die Verordnung von Innovationen resultierte, die bei Berechnung der Richtgröße noch nicht angesetzt werden konnten. Hiermit hat sich allerdings, so weit ersichtlich, die Rechtsprechung noch nicht auseinander gesetzt.

\section{Praxisbesonderheiten gemäß Anlage}

Darüber hinaus können im Richtgrößenprüfverfahren auch Verordnungskosten als Praxisbesonderheiten geltend gemacht werden, die sich aus den Anlagen zur Prüfvereinbarung/Richtgrößenvereinbarung ergeben. Dabei sind regional unterschiedlich in den genannten Vereinbarungen Wirkstoffe und Indikationen gelistet, die von den Prüfgremien als Praxisbesonderheiten berücksichtigt werden sollen. Eine Rahmenempfehlung auf Bundesebene sah hierzu sogar vor, dass die Kosten dieser Medikamente vor Beginn der Richtgrößenprüfung aus dem Verordnungsvolumen des Arztes herausgerechnet werden sollten. In zahlreichen KV-Bezirken sollen daher Listen mit den Verordnungsdaten und -summen bestimmter Arzneimittel bereits vor Beginn eines Prüfverfahrens eingereicht werden, damit das behauptete Verordnungsvolumen entsprechend bereinigt werden kann. Die Erfahrung zeigt überdies, dass regional unterschiedlich auf diesem Wege auch Arzneimittelkosten berücksichtigt und in Abzug gebracht werden, die zwar nicht in der jeweiligen Anlage zur jeweils gültigen Richtgrößenvereinbarung gelistet sind, über deren Anerkennungsfähigkeit aber seitens der Prüfgremien Einigkeit besteht.

Für die Argumentation im Prüfverfahren ist die genaue Kenntnis solcher Anlagen zu den Prüfvereinbarungen/Richtgrößenvereinbarungen unerlässlich. Beispielsweise nach der Anlage D zur Richtgrößenvereinbarung 2004 der KV Nordrhein gilt die „systemische Psoriasistherapie“ als eine solche Praxisbesonderheit. Hiermit lässt sich auch in solchen KV-Bezirken argumentieren, in denen eine Aufnahme in die Anlage noch nicht vereinbart werden konnte.

\section{Literatur}

\footnotetext{
${ }^{1}$ BSG SozR 3-2500 §106 Nr. 27: 150-153

2 BSG MedR 1996: 138f.

${ }^{3}$ Engelhard W. In: Hauck K. SGB V, Kommentar, §106 Rdnr. 98

${ }^{4}$ GKV-GRG 2000, BT-Drucks. 14/1245: S. 81 zu $§ 106$ Abs. 5

${ }^{5}$ Engelhard W. In: Hauck K. SGB V, Kommentar, § 106 Rdnr. 98 e

${ }^{6}$ Clemens T. In: Schulin B (Hrsg). Handbuch des Sozialversicherungsrechts, Bd. 1, §35 Rz. 83)
}

Lokaltherapeutische Standards für chronische Hautwunden. Ulcus cruris, Dekubitus, Kompressionstherapie, Weichlagerung G. Kammerlander

Wien: Springer, 3. Aufl. 2005, 296 S. mit Ergänzungsheft, 607 meist farb. Abb., 59,80€, ISBN 3-211-22472-6

Das Thema chronische Wunden und Wundheilung findet in den letzten Jahren wiederholt ihren Niederschlag auch in deutschsprachigen Monografien und ist Ausdruck eines allgemein zunehmenden Interesses sowohl von Seiten der Ärzte als auch des medizinischen Pflegepersonals. Das vorliegende Buch von Herrn Kammerlander zeichnet sich dabei in mehrfacher Weise aus: 1. Es entstammt jahrelanger eigener Praxis eines dem Thema leidenschaftlich zugewandten dermatologischen Pflegers mit eindrucksvoller Vita (u.a. Vizepräsident dreier Fachgesellschaften für Wundheilung in Deutschland, Österreich und in der Schweiz). 2. Es nennt nahezu alle derzeit auf dem Markt verfügbaren Produkte zur Wundheilung und beschreibt bis ins Detail deren Anwendungseigenschaften, Indikation und Kontraindikationen. 3. Es ist didaktisch hervorragend bebildert und mit umfangreichen, zumeist übersichtlichen Tabellen ausgestattet.

Das Buch ist kein Lehrbuch und wirkt nicht aus einem Guss. Es entstammt, wie der Autor selbst anführt, einer jahrelangen Sammlung aller ihm zugänglichen Informationen, in vielen Fällen der Industrie mit ihren Produkten zur Prophylaxe und Therapie chronischer Wunden, aber auch zahlreicher dermatologischer und chirurgischer Lehrbücher sowie Artikel in Fachzeitschriften, national wie international. Redundanzen in der Information sind bei diesem Vorgehen nicht zu vermeiden, stören aber nicht, da sie den gelernten Wissensstoff festigen. Ärzte und Pflegepersonal ziehen praktischen Gewinn aus einem detaillierten Nachschlagewerk zur Versorgung chronischer Wunden, besonders des Ulcus cruris und des Dekubitalulcus, der Kompressionstherapie und der Weichlagerung von Wunden. Um up to date zu bleiben, erscheinen Neuentwicklungen der Wundversorgung in einem eigenen Ergänzungsheft, das dem Buch beigefügt wird. Das Buch macht auch den auf dem Gebiet chronischer Wunden Erfahrenen neugierig und sollte sowohl im Krankenhaus als auch in der Praxis Ärzten und Pflegepersonal jederzeit zugänglich sein.

M. Buslau, Bad Säckingen 